\title{
Families of $v$-similar birth-death processes and limiting conditional distributions
}

\section{Anna Poskrobko* and Ewa Girejko}

\section{"Correspondence:}

a.poskrobko@pb.edu.pl

Faculty of Computer Science,

Bialystok University of Technology,

ul. Wiejska 45A, Białystok, 15-351,

Poland

\begin{abstract}
We study, for a given process $\mathcal{X}$, the conditions under which $\mathcal{v}$-similarity is provided, relations between similarity and $\boldsymbol{\nu}$-similarity of processes, and we analyze (doubly) limiting conditional distributions. We present sufficient conditions for the existence of at least one $\boldsymbol{v}$-similar process to a given one. Moreover, recursive formulas for birth and death rates of $\boldsymbol{v}$-similar processes are formulated. We apply differential equations and uniqueness of solutions to such equations to derive the main results of the paper. Finally, an example illustrates the applications of our results.
\end{abstract}

MSC: Primary 60J80; 42C05; secondary 60J85

Keywords: birth-death process; $v$-similarity; limiting conditional distribution

\section{Introduction}

In this paper we are concerned with the concept of $v$-similarity for birth-death processes. The idea of similarity was introduced by Di Crescenzo in 1994 (see [1]). Two birth-death processes $\mathcal{X}$ and $\widetilde{\mathcal{X}}$ are said to be similar if their transition probability functions $p$ and $\tilde{p}$ satisfy

$$
\tilde{p}_{i j}(t)=\frac{v_{j}}{v_{i}} p_{i j}(t)
$$

where $v_{i}, v_{j}$ are constants.

Later on the theme of similar processes has been investigated by some authors in the last two decades. Therefore, for one-dimensional birth-death processes it was considered by Lenin et al. [2], who define the more general idea of introducing the notion of similarity constants $c_{i j}$,

$$
\tilde{p}_{i j}(t)=c_{i j} p_{i j}(t)
$$

showing that $c_{i j}=\alpha_{i} \beta_{j}$; for Markov chains by Pollett [3], who calls such a concept of similarity a strong similarity; for random walks by Schiefermayr [4]. Several authors extended their results to the two-dimensional case [5], others investigated birth-death processes with killing [6-8].

In [2] the authors consider also the concept of $v$-similarity,

$$
\tilde{p}_{i j}(t)=c_{i j} e^{v t} p_{i j}(t)
$$

(C) 2015 Poskrobko and Girejko. This article is distributed under the terms of the Creative Commons Attribution 4.0 International License (http://creativecommons.org/licenses/by/4.0/), which permits unrestricted use, distribution, and reproduction in any medium, provided you give appropriate credit to the original author(s) and the source, provide a link to the Creative Commons license, and indicate if changes were made. 
and derive the necessary condition for the existence of distinct $v$-similar birth-death processes.

Moreover, in [9] and [10] the authors study the spectral structure of birth-death processes and present simple conditions for the classification of boundaries, while in [11] a bilateral birth-death process that is similar to the birth-death process over $\mathbb{Z}$ with constant rates is considered.

In our work we extend these results and formulate the sufficient condition for the existence of $v$-similar processes and specify a one-parameter family of birth-death processes $v$-similar to a given one. It is well known that the time-dependent behavior of birth-death processes involves orthogonal polynomials and, therefore, its orthogonalizing measure. Our purpose is to study, using orthogonal polynomials, the conditions under which $v$-similarity is provided, relations between similarity and $v$-similarity of processes and to analyze (doubly) limiting conditional distributions. Such distributions, as Schoutens states in [12], can be used to gain insight into the asymptotic behavior of processes with absorbing and reflecting states. In order to derive sufficient conditions for the existence of at least one $v$-similar process to a given one, we use differential equations and uniqueness of solutions to such equations.

The paper is organized as follows. In Section 2 preliminary definitions and notions are gathered. Section 3 is the one that contains main results, namely Theorem 10, which gives a sufficient condition for the existence of $v$-similar process to the given one, and Theorem 11, which provides necessary and sufficient conditions for the existence of $v$-similar processes to the given one with recursive formulas for birth and death rates. Further, we give the formulas of the limiting conditional distribution and the doubly limiting conditional distribution for a $v$-similar process. We end the paper presenting an illustrative example.

\section{Preliminaries}

Let $\mathcal{X}=\{X(t), t \geq 0\}$ denote a birth-death process on the state space $\mathcal{S}=\mathcal{N} \cup\{-1\}$, where $\mathcal{N}=\{0,1,2, \ldots\}$ with transition probability function

$$
p_{i j}(t)=\operatorname{Pr}[X(t)=j \mid X(0)=i], \quad i, j \in \mathcal{S}, t \geq 0 .
$$

We assume that the transition functions $p_{i j}(t)$ satisfy both the Kolmogorov backward equation

$$
p_{i j}^{\prime}(t)=\sum_{k \in \mathcal{S}} q_{i k} p_{k j}(t), \quad i, j \in \mathcal{S}, t \geq 0,
$$

and the forward equation

$$
p_{i j}^{\prime}(t)=\sum_{k \in \mathcal{S}} p_{i k}(t) q_{k j}, \quad i, j \in \mathcal{S}, t \geq 0,
$$

where $q_{i, i+1}=\lambda_{i}, q_{i, i-1}=\mu_{i}$, and $q_{i i}=-\left(\lambda_{i}+\mu_{i}\right)$ for all $i \in \mathcal{N}$ and $q_{i j}=0$ otherwise. The constants $\lambda_{j}$ (birth rates) and $\mu_{j}$ (death rates) may be thought of as the rates of absorption from state $j$ into states $j+1$ and $j-1$, respectively, with $\lambda_{j}>0, \mu_{j+1}>0, j \in \mathcal{N}, \mu_{0} \geq 0$. Additionally, we assume that the potential coefficients

$$
\pi_{0}=1, \quad \pi_{j}=\frac{\lambda_{0} \lambda_{1} \cdots \lambda_{j-1}}{\mu_{1} \mu_{2} \cdots \mu_{j}}, \quad j>0,
$$


satisfy the condition

$$
\sum_{j=0}^{\infty}\left(\pi_{j}+\left(\lambda_{j} \pi_{j}\right)^{-1}\right)=\infty .
$$

Karlin and McGregor [13] have shown that the transition probabilities $p_{i j}(t)$ can be represented as

$$
p_{i j}(t)=\pi_{j} \int_{0}^{\infty} e^{-x t} Q_{i}(x) Q_{j}(x) d \varrho(x) .
$$

By $\left\{Q_{j}(x)\right\}$ we mean a sequence of birth-death polynomials defined recursively as follows:

$$
\begin{aligned}
& Q_{-1}(x)=0, \quad Q_{0}(x)=1, \\
& -x Q_{j}(x)=\mu_{j} Q_{j-1}(x)-\left(\lambda_{j}+\mu_{j}\right) Q_{j}(x)+\lambda_{j} Q_{j+1}(x), \quad j \geq 1,
\end{aligned}
$$

orthogonal with respect to the spectral measure @, i.e.

$$
\pi_{j} \int_{0}^{\infty} Q_{i}(x) Q_{j}(x) d \varrho(x)=\delta_{i j}
$$

where $\delta_{i j}$ denotes the Kronecker delta. It was shown by Karlin and McGregor [13] that there is at least one such measure with total mass 1 on $[0, \infty)$.

Remark 1 The polynomial $Q_{j}(x), j>0$ has $j$ positive distinct zeros $x_{j 1}<x_{j 2}<\cdots<x_{j j}$ and its leading coefficient equals $\frac{(-1)^{j}}{\lambda_{0} \lambda_{1} \cdots \lambda_{j-1}}, j>0$. Hence $Q_{j}(x)>0$ for $x \leq \xi_{1}$, where $\xi_{1} \equiv \lim _{j \rightarrow \infty} x_{j 1}$ with $0 \leq \xi_{1}<x_{j 1}$ for $j>0$ (see [14]).

Let us consider the series

$$
\begin{aligned}
& A=\sum_{j=0}^{\infty} \frac{1}{\lambda_{j} \pi_{j}}, \quad B=\sum_{j=0}^{\infty} \pi_{j}, \\
& C=\sum_{j=0}^{\infty} \frac{1}{\lambda_{j} \pi_{j}} \sum_{i=0}^{j} \pi_{i}, \quad D=\sum_{j=0}^{\infty} \frac{1}{\lambda_{j} \pi_{j}} \sum_{i=j+1}^{\infty} \pi_{i} .
\end{aligned}
$$

We follow the notation of [15] and [16]. The series $C$ and $D$ determine the behavior of the process $\mathcal{X}$ near the boundary point at infinity. The assumptions $C=\infty$ and $D=\infty$ rule out the possibility of an explosion of the process $\mathcal{X}$, i.e. reaching infinity in finite time. Although $A$ and $B$ have no physical interpretation, they are related to $C$ and $D$, i.e. $C+D=A B$ and $A+B=\infty$ (which is equivalent to (1)) if and only if at least one of $C$ or $D$ diverges. The divergence of the series (3) guarantees the convergence of the probability to a proper limiting and doubly limiting conditional distributions. Let us consider conditional probabilities of the form

$$
\begin{aligned}
& r_{i j}(t)=\operatorname{Pr}[X(t)=j \mid X(0)=i, X(t) \in \mathcal{N}, X(t+\sigma)=-1 \text { for some } \sigma>0], \\
& r_{i j}(t, s)=\operatorname{Pr}[X(t)=j \mid X(0)=i, X(t) \in \mathcal{N}, X(t+s+\sigma)=-1 \text { for some } \sigma>0]
\end{aligned}
$$


for a process with absorbing state $\left(\mu_{0}>0\right)$ and

$$
\begin{aligned}
& r_{i j}(t)=\operatorname{Pr}[X(t)=j \mid X(0)=i, X(t+\sigma)=0 \text { for some } \sigma>0], \\
& r_{i j}(t, s)=\operatorname{Pr}[X(t)=j \mid X(0)=i, X(t+s+\sigma)=0 \text { for some } \sigma>0]
\end{aligned}
$$

in the irreducible case $\left(\mu_{0}=0\right)$. Here $i, j \in \mathcal{N}, t \geq 0$. It was proved by Kijima et al. [16] and Schoutens [12] that, for the absorbing case, if $C=\infty$ and $D=\infty$ then the limiting conditional distribution and the doubly limiting conditional distribution of the process $\mathcal{X}$ are given by

$$
\begin{aligned}
& \lim _{t \rightarrow \infty} r_{i j}(t)=\frac{\pi_{j} Q_{j}\left(\xi_{1}\right)}{\sum_{k=0}^{\infty} \pi_{k} Q_{k}\left(\xi_{1}\right)}, \\
& \lim _{t \rightarrow \infty} \lim _{s \rightarrow \infty} r_{i j}(t, s)=\frac{\pi_{j} Q_{j}^{2}\left(\xi_{1}\right)}{\sum_{k=0}^{\infty} \pi_{k} Q_{k}^{2}\left(\xi_{1}\right)} .
\end{aligned}
$$

For the reflecting case $\mu_{0}=0$, if $B=\infty$ and $C=\infty(B=\infty$ implies $D=\infty)$ then

$$
\begin{aligned}
& \lim _{t \rightarrow \infty} r_{i j}(t)=\frac{a_{j} \pi_{j} Q_{j}\left(\xi_{1}\right)}{\sum_{k=0}^{\infty} a_{k} \pi_{k} Q_{k}\left(\xi_{1}\right)}, \\
& \lim _{t \rightarrow \infty} \lim _{s \rightarrow \infty} r_{i j}(t, s)=\frac{\pi_{j} Q_{j}^{2}\left(\xi_{1}\right)}{\sum_{k=0}^{\infty} \pi_{k} Q_{k}^{2}\left(\xi_{1}\right)},
\end{aligned}
$$

where

$$
a_{j}=\frac{\int_{0}^{\infty} x^{-1} Q_{j}(x) d \varrho(x)}{\int_{0}^{\infty} x^{-1} d \varrho(x)} .
$$

The above limits equal 0 whenever the series in the denominators diverge. We refer the reader to [16] for a detailed discussion of the limiting conditional distribution problem.

Besides $\mathcal{X}$, let us consider another process $\tilde{\mathcal{X}}$, determined by birth rates $\tilde{\lambda}_{j}$ and death rates $\tilde{\mu}_{j}$ with potential coefficients $\tilde{\pi}_{j}$ and transition functions $\tilde{p}_{i j}(t)$.

Definition 2 ([2]) The birth-death processes $\mathcal{X}$ and $\widetilde{\mathcal{X}}$ are said to be similar if the transition probability functions of $\mathcal{X}$ and $\widetilde{\mathcal{X}}$ differ only by a constant factor, that is, if there are constants $c_{i j}>0, i, j \in \mathcal{N}$ such that

$$
\tilde{p}_{i j}(t)=c_{i j} p_{i j}(t), \quad t \geq 0 .
$$

For the classical works on similar birth-death processes, see [1] and [2]. It is well known that if $\mathcal{X}$ and $\tilde{\mathcal{X}}$ are similar processes, then their birth and death rates are related as follows: $\tilde{\lambda}_{j}+\tilde{\mu}_{j}=\lambda_{j}+\mu_{j}$ and $\tilde{\lambda}_{j} \tilde{\mu}_{j+1}=\lambda_{j} \mu_{j+1}, j \in \mathcal{N}$, with their transition functions satisfying $\tilde{p}_{i j}(t)=\sqrt{\frac{\pi_{i} \tilde{\pi}_{j}}{\tilde{\pi}_{i} \pi_{j}}} p_{i j}(t), i, j \geq 0, t \geq 0$. The converse statement is true only under an additional condition. Indeed, if

$$
\sum_{j=1}^{\infty} \pi_{j}\left(\sum_{k=0}^{j-1}\left(\lambda_{k} \pi_{k}\right)^{-1}\right)^{2}=\infty
$$


for the process $\mathcal{X}$ with $\mu_{0}>0$ and $\tilde{\mathcal{X}}$ is a birth-death process the rates of which are related to those of $\mathcal{X}$ as $\tilde{\lambda}_{j}+\tilde{\mu}_{j}=\lambda_{j}+\mu_{j}$ and $\tilde{\lambda}_{j} \tilde{\mu}_{j+1}=\lambda_{j} \mu_{j+1}, j \in \mathcal{N}$, then the processes $\widetilde{\mathcal{X}}$ and $\mathcal{X}$ are similar. In the sequel we will assume the validity of condition (4) (which is stronger than (1)), when $\mu_{0}>0$.

Reference [2] gives the conditions under which the phenomenon of similarity occurs. It also identifies the family of all processes which are similar to a given process.

Theorem 3 ([2]) A birth-death process $\mathcal{X}$ with birth rates $\left\{\lambda_{j}: j \in \mathcal{N}\right\}$ and death rates $\left\{\mu_{j}: j \in \mathcal{N}\right\}$ is not similar to any other birth-death process if and only if

$$
\mu_{0}=0 \quad \text { and } \quad \sum_{j=0}^{\infty} \frac{1}{\lambda_{j} \pi_{j}}=\infty .
$$

In the opposite case the process is similar to any member of an infinite, one-parameter family of birth-death processes $\left\{\widetilde{\mathcal{X}}^{(x)}: 0 \leq x \leq 1 / S\right\}$ if $\mu_{0}=0$, and $\left\{\widetilde{\mathcal{X}}^{(x)}:-\infty \leq x \leq 1 / T\right\}$ if $\mu_{0}>0$. The birth rates $\tilde{\lambda}_{j}^{(x)}$ and death rates $\tilde{\mu}_{j}^{(x)}, j \in \mathcal{N}$, of $\tilde{\mathcal{X}}^{(x)}$ are given by

$$
\tilde{\mu}_{0}^{(x)} \equiv \lambda_{0} x, \quad \tilde{\lambda}_{j}^{(x)}=\lambda_{j} \frac{1-x S_{j}}{1-x S_{j-1}}, \quad \tilde{\mu}_{j+1}^{(x)}=\mu_{j+1} \frac{1-x S_{j-1}}{1-x S_{j}}, \quad j \geq 0,
$$

if $\mu_{0}=0$, and

$$
\tilde{\lambda}_{j}^{(x)}=\lambda_{j} \frac{1-x T_{j+1}}{1-x T_{j}}, \quad \tilde{\mu}_{j}^{(x)}=\mu_{j} \frac{1-x T_{j-1}}{1-x T_{j}}, \quad j \geq 0,
$$

if $\mu_{0}>0$. Here $S_{j}=\lambda_{0} \sum_{k=0}^{j}\left(\lambda_{k} \pi_{k}\right)^{-1}, S_{-1} \equiv 0, S=\lim _{j \rightarrow \infty} S_{j}$, and $T_{j}=\mu_{0} \sum_{k=0}^{j}\left(\mu_{k} \pi_{k}\right)^{-1}$, $T_{-1} \equiv 0, T=\lim _{j \rightarrow \infty} T_{j}$.

Remark 4 The similarity constants $C_{i j}^{(x)}, i, j \geq 0$, for processes $\mathcal{X}$ and $\widetilde{\mathcal{X}}^{(x)}$ equal $C_{i j}^{(x)}=$ $\frac{1-x S_{j-1}}{1-x S_{i-1}}$ if $\mu_{0}=0$ and $C_{i j}^{(x)}=\frac{1-x T_{j}}{1-x T_{i}}$ if $\mu_{0}>0$.

Remark 5 Let us notice that if the process $\mathcal{X}$ is positive recurrent (or ergodic) and the processes $\mathcal{X}$ and $\tilde{\mathcal{X}}$ are similar, then also the process $\tilde{\mathcal{X}}$ (with $\tilde{\mu}_{0}=0$ ) is positive recurrent (ergodic).

\section{Processes $v$-similar}

In this section we present our main results that concern conditions providing existence of $v$-similar family of processes to the given birth-death process.

Definition 6 ([2]) The birth-death process $\widetilde{\mathcal{X}}$ is said to be $v$-similar to the birth-death process $\mathcal{X}$ for some real number $v$ if there are constants $c_{i j}>0, i, j \in \mathcal{N}$, such that

$$
\tilde{p}_{i j}(t)=c_{i j} e^{\nu t} p_{i j}(t), \quad t \geq 0 .
$$

See Lenin et al. [2] for more details. For the special case $v=0$, we say $\tilde{\mathcal{X}}$ is similar to $\mathcal{X}$. The relation ' $v$-similar' is an equivalence relation if $v=0$ but not if $v \neq 0$.

We mention some results concerning $v$-similarity of birth-death processes (see [17] and [2]). 
Theorem 7 ([17]) If $\mathcal{X}$ and $\tilde{\mathcal{X}}$ are birth-death processes such that $\tilde{\mathcal{X}}$ is $v$-similar, $v \leq \xi_{1}$ to $\mathcal{X}$, then their distribution functions of the spectral measures satisfy $\tilde{\varrho}(x)=\varrho(x+v), x \geq 0$.

Theorem 8 ([2]) If a birth-death process $\widetilde{\mathcal{X}}$ is $v$-similar to a birth-death process $\mathcal{X}$, then their birth and death rates are related as

$$
\tilde{\lambda}_{j}+\tilde{\mu}_{j}=\lambda_{j}+\mu_{j}-v, \quad \tilde{\lambda}_{j} \tilde{\mu}_{j+1}=\lambda_{j} \mu_{j+1}, \quad \text { where } j \in \mathcal{N},
$$

with their transition functions satisfying $\tilde{p}_{i j}(t)=\sqrt{\frac{\pi_{i} \tilde{\pi}_{j}}{\tilde{\pi}_{i} \pi_{j}}} e^{\nu t} p_{i j}(t), i, j \geq 0, t \geq 0$.

Theorem 9 ([2]) For each $v \leq \xi_{1}$ there is a unique solution $\tilde{\lambda}_{j}=\tilde{\lambda}_{j}^{(v)}$ and $\tilde{\mu}_{j}=\tilde{\mu}_{j}^{(v)}, j \in \mathcal{N}$, to $(7)$ satisfying $\tilde{\mu}_{0}=0$ which is given by $\tilde{\lambda}_{j}=\lambda_{j} \frac{Q_{j+1}(v)}{Q_{j}(v)}, \tilde{\mu}_{j+1}=\mu_{j+1} \frac{Q_{j}(v)}{Q_{j+1}(v)}, j \in \mathcal{N}$. There is no solution to the system (7) if $v>\xi_{1}$.

In a comparable manner to the notion of similarity, the converse statement to Theorem 8 is also true only under condition (4).

Theorem 10 Let $\mathcal{X}$ be a birth-death process that satisfies (4) if $\mu_{0}>0$, and let $\tilde{\mathcal{X}}$ be a birth-death process with the rates related to those of $\mathcal{X}$, satisfying equations (7) for some real $v$. Then the process $\tilde{\mathcal{X}}$ is $v$-similar to $\mathcal{X}$.

Proof The concept of the proof is adapted from [2] where the case $v=0$ is considered. Let us consider the matrices

$$
\begin{aligned}
& P(t)=\left(p_{i j}(t)\right)_{i, j \in \mathcal{N}^{\prime}}, \quad t \geq 0, \\
& Q=\left(\begin{array}{cccccc}
-\left(\lambda_{0}+\mu_{0}\right) & \lambda_{0} & 0 & 0 & 0 & \ldots \\
\mu_{1} & -\left(\lambda_{1}+\mu_{1}\right) & \lambda_{1} & 0 & 0 & \ldots \\
0 & \mu_{2} & -\left(\lambda_{2}+\mu_{2}\right) & \lambda_{2} & 0 & \ldots \\
\vdots & \vdots & \vdots & \vdots & \vdots & \ddots
\end{array}\right), \\
& A=\left(\begin{array}{cccccc}
-\left(\lambda_{0}+\mu_{0}\right) & \sqrt{\lambda_{0} \mu_{1}} & 0 & 0 & 0 & \ldots \\
\sqrt{\lambda_{0} \mu_{1}} & -\left(\lambda_{1}+\mu_{1}\right) & \sqrt{\lambda_{1} \mu_{2}} & 0 & 0 & \ldots \\
0 & \sqrt{\lambda_{1} \mu_{2}} & -\left(\lambda_{2}+\mu_{2}\right) & \sqrt{\lambda_{2} \mu_{3}} & 0 & \ldots \\
\vdots & \vdots & \vdots & \vdots & \vdots & \ddots
\end{array}\right) .
\end{aligned}
$$

The matrix $A$ can be represented as

$$
A=\Pi^{\frac{1}{2}} Q \Pi^{-\frac{1}{2}}
$$

where $\Pi^{1 / 2}$ and $\Pi^{-1 / 2}$ denote the diagonal matrices with entries $\pi_{j}^{1 / 2}$ and $\pi_{j}^{-1 / 2}, j \in \mathcal{N}$, respectively, on the diagonals. It follows that we can write the Kolmogorov backward and forward equations in the form

$$
\Pi^{\frac{1}{2}} P^{\prime}(t) \Pi^{-\frac{1}{2}}=A \Pi^{\frac{1}{2}} P(t) \Pi^{-\frac{1}{2}}=\Pi^{\frac{1}{2}} P(t) \Pi^{-\frac{1}{2}} A, \quad t \geq 0 .
$$

Denoting

$$
R(t)=\Pi^{\frac{1}{2}} P(t) \Pi^{-\frac{1}{2}}, \quad t \geq 0,
$$


we can rewrite (9) as

$$
\left\{\begin{array}{l}
R^{\prime}(t)=A R(t)=R(t) A, \quad t \geq 0 \\
R(0)=I
\end{array}\right.
$$

where $I$ denotes the infinite identity matrix.

If the birth and death rates of $\mathcal{X}$ and $\widetilde{\mathcal{X}}$ are related as in (7) we have

$$
\widetilde{A}=\widetilde{\Pi}^{\frac{1}{2}} \widetilde{Q} \widetilde{\Pi}^{-\frac{1}{2}}=A+v I
$$

It turns out that $\widetilde{R}(t)=\widetilde{\Pi}^{1 / 2} \widetilde{P}(t) \widetilde{\Pi}^{-1 / 2}$ is a solution to the system

$$
\left\{\begin{array}{l}
\widetilde{R}^{\prime}(t)=(A+v I) \widetilde{R}(t)=\widetilde{R}(t)(A+v I), \quad t \geq 0 \\
\widetilde{R}(0)=I
\end{array}\right.
$$

If the solution of (10) is unique then we have the following unique solution to (11):

$$
\widetilde{R}(t)=e^{v t} R(t)
$$

Therefore

$$
\widetilde{\Pi} \frac{1}{2} \widetilde{P}(t) \widetilde{\Pi}^{-\frac{1}{2}}=e^{v t} \Pi^{\frac{1}{2}} P(t) \Pi^{-\frac{1}{2}}
$$

and

$$
\widetilde{P}(t)=e^{v t} \widetilde{\Pi}^{-\frac{1}{2}} \Pi^{\frac{1}{2}} P(t) \Pi^{-\frac{1}{2}} \widetilde{\Pi}^{\frac{1}{2}}
$$

Thus processes $\mathcal{X}$ and $\tilde{\mathcal{X}}$ are $v$-similar. There is a unique (relevant) solution to (10) if and only if either $\mu_{0}=0$ and (1) holds true or $\mu_{0}>0$ and (4) holds true (see [2]).

Theorem 11 Let $\mathcal{X}$ be the birth-death process with birth rates $\left\{\lambda_{j}, j \in \mathcal{N}\right\}$ and death rates $\left\{\mu_{j}, j \in \mathcal{N}\right\}$ and let $v \leq \xi_{1}$. Then there exists a birth-death process $\widetilde{\mathcal{X}} \neq \mathcal{X}$ which is $v$-similar to $\mathcal{X}$ if and only if

$$
\sum_{j=0}^{\infty} \frac{1}{\lambda_{j} \pi_{j} Q_{j}(v) Q_{j+1}(v)}<\infty \text { and } \sum_{j=0}^{\infty} \pi_{j} Q_{j}^{2}(v)=\infty
$$

If (12) holds, then any member of an infinite, one-parameter family of the birth-death processes $\left\{\widetilde{\mathcal{X}}^{(x, v)}: 0 \leq x \leq 1 / S^{(v)}\right\}$ is $v$-similar to $\mathcal{X}$, where the parameters $\left\{\tilde{\lambda}_{j}^{(x, v)}, \tilde{\mu}_{j}^{(x, v)}\right\}_{j=0}^{\infty}$ of $\widetilde{\mathcal{X}}^{(x, v)}$ are given by

$$
\begin{aligned}
& \tilde{\lambda}_{j}^{(x, v)}=\frac{Q_{j+1}(v)}{Q_{j}(v)} \lambda_{j} \frac{1-x S_{j}^{(v)}}{1-x S_{j-1}^{(v)}}, \\
& \tilde{\mu}_{j+1}^{(x, v)}=\frac{Q_{j}(v)}{Q_{j+1}(v)} \mu_{j+1} \frac{1-x S_{j-1}^{(v)}}{1-x S_{j}^{(v)}}, \\
& \tilde{\mu}_{0}^{(x, v)}=\left(\lambda_{0}+\mu_{0}-v\right) x,
\end{aligned}
$$


where $S_{-1}^{(\nu)}=0$,

$$
S_{j}^{(v)}=\left(\lambda_{0}+\mu_{0}-v\right) \sum_{k=0}^{j} \frac{1}{\lambda_{k} \pi_{k} Q_{k}(v) Q_{k+1}(v)},
$$

and $S^{(v)}=\lim _{j \rightarrow \infty} S_{j}^{(v)}$. The similarity constants $C_{i j}^{(x, v)}, i, j \geq 0$, are given by

$$
C_{i j}^{(x, v)}=\frac{Q_{j}(v)}{Q_{i}(v)} \cdot \frac{1-x S_{j-1}^{(v)}}{1-x S_{i-1}^{(\nu)}} .
$$

Proof By Theorem 9 there exists a unique birth-death process $\widehat{\mathcal{X}}$ with parameters $\hat{\lambda}_{j}, \hat{\mu}_{j}$, $j \in \mathcal{N}$, and $\hat{\mu}_{0}=0$ which is $v$-similar to $\mathcal{X}$ for $v \leq \xi_{1}$. Using the equalities from Theorem 9 it is easily seen that $\hat{\pi}_{j}=\pi_{j} Q_{j}^{2}(v)$ and

$$
\sum_{j=0}^{\infty} \frac{1}{\hat{\lambda}_{j} \hat{\pi}_{j}}=\sum_{j=0}^{\infty} \frac{1}{\lambda_{j} \pi_{j} Q_{j}(v) Q_{j+1}(v)} .
$$

We know from Theorem 3 that there exists a process $\widetilde{\mathcal{X}}$ with $\tilde{\mu}_{0}>0$ similar to $\widehat{\mathcal{X}}$ if and only if $\sum_{j=0}^{\infty} \frac{1}{\hat{\lambda}_{j} \hat{\pi}_{j}}<\infty$ and $\sum_{j=0}^{\infty}\left(\hat{\pi}_{j}+\left(\hat{\lambda}_{j} \hat{\pi}_{j}\right)^{-1}\right)=\infty$. By the above, the process $\tilde{\mathcal{X}} \neq \mathcal{X}$, with $\tilde{\mu}_{0}>0$, is $\nu$-similar to the process $\mathcal{X}$ if and only if

$$
\sum_{j=0}^{\infty} \frac{1}{\lambda_{j} \pi_{j} Q_{j}(v) Q_{j+1}(v)}<\infty \quad \text { and } \quad \sum_{j=0}^{\infty} \pi_{j} Q_{j}^{2}(v)=\infty .
$$

By Theorem 3 and Theorem 9 it follows that any member of the one-parameter family $\widetilde{\mathcal{X}}^{(x, v)}$ is $v$-similar to $\mathcal{X}$ when $0 \leq x \leq 1 / S^{(v)}, S^{(v)}=\lim _{j \rightarrow \infty} S_{j}^{(v)}$, and

$$
\begin{aligned}
S_{j}^{(v)} & =\hat{\lambda}_{0} \sum_{k=0}^{j} \frac{1}{\hat{\lambda}_{k} \hat{\pi}_{k}}=\lambda_{0} Q_{1}(v) \sum_{k=0}^{j} \frac{1}{\lambda_{k} \pi_{k} Q_{k}(v) Q_{k+1}(v)} \\
& =\left(\lambda_{0}+\mu_{0}-v\right) \sum_{k=0}^{j} \frac{1}{\lambda_{k} \pi_{k} Q_{k}(v) Q_{k+1}(v)} \\
\tilde{\lambda}_{j}^{(x, v)} & =\hat{\lambda}_{j} \frac{1-x S_{j}^{(v)}}{1-x S_{j-1}^{(v)}}=\lambda_{j} \frac{Q_{j+1}(v)}{Q_{j}(v)} \cdot \frac{1-x S_{j}^{(v)}}{1-x S_{j-1}^{(v)}} \\
\tilde{\mu}_{j+1}^{(x, v)} & =\hat{\mu}_{j+1} \frac{1-x S_{j-1}^{(v)}}{1-x S_{j}^{(v)}}=\mu_{j+1} \frac{Q_{j}(v)}{Q_{j+1}(v)} \cdot \frac{1-x S_{j-1}^{(v)}}{1-x S_{j}^{(v)}} \\
\tilde{\mu}_{0}^{(x, v)} & =\hat{\lambda}_{0} x=\lambda_{0} Q_{1}(v) x=\left(\lambda_{0}+\mu_{0}-v\right) x .
\end{aligned}
$$

It is easily seen that the constants $\tilde{\pi}_{j}^{(x, v)}=\frac{\tilde{\lambda}_{0}^{(x, v)} \ldots \tilde{\lambda}_{j-1}^{(x, v)}}{\tilde{\mu}_{1}^{(x, v)} \ldots \tilde{\mu}_{j}^{(x, v)}}$ satisfy $\tilde{\pi}_{j}^{(x, v)}=\pi_{j} Q_{j}^{2}(v)\left(1-x S_{j-1}^{(\nu)}\right)^{2}$. Hence (14) is an immediate consequence of Theorem 3.

\section{Corollary 12}

(i) If $\mu_{0}>0$, for $\nu \leq 0$, the condition (12) holds.

(ii) If $\mu_{0}=0$ and $\sum_{j=0}^{\infty} \frac{1}{\lambda_{j} \pi_{j}}<\infty, \sum_{j=0}^{\infty} \pi_{j}=\infty$, for $v \leq 0$, the condition (12) holds. 
Proof (i) Let $\tilde{\mathcal{X}}$ be the birth-death process with parameters $\tilde{\lambda}_{j}, \tilde{\mu}_{j}, j \geq 0$ defined by $\tilde{\mu}_{0}=0$, $\tilde{\lambda}_{j}=\frac{Q_{j+1}(0)}{Q_{j}(0)} \lambda_{j}, \tilde{\mu}_{j+1}=\frac{Q_{j}(0)}{Q_{j+1}(0)} \mu_{j+1}$ for $j \geq 0$. Then, by Theorem 9, $\tilde{\mathcal{X}}$ is similar to $\mathcal{X}$.

Since $\mathcal{X}$ is similar to $\widetilde{\mathcal{X}}$, by Theorem 3 and (1) we get

$$
\sum_{j=0}^{\infty} \frac{1}{\tilde{\lambda}_{j} \tilde{\pi}_{j}}=\sum_{j=0}^{\infty} \frac{1}{\lambda_{j} \pi_{j} Q_{j}(0) Q_{j+1}(0)}<\infty
$$

and

$$
\sum_{j=0}^{\infty} \tilde{\pi}_{j}=\sum_{j=0}^{\infty} \pi_{j} Q_{j}^{2}(0)=\infty
$$

And now, by Remark $1, v \leq 0$ implies $Q_{j}(v) \geq Q_{j}(0)$,

$$
\begin{aligned}
& \sum_{j=0}^{\infty} \frac{1}{\lambda_{j} \pi_{j} Q_{j}(v) Q_{j+1}(v)} \leq \sum_{j=0}^{\infty} \frac{1}{\lambda_{j} \pi_{j} Q_{j}(0) Q_{j+1}(0)}, \\
& \sum_{j=0}^{\infty} \pi_{j} Q_{j}^{2}(0) \leq \sum_{j=0}^{\infty} \pi_{j} Q_{j}^{2}(v),
\end{aligned}
$$

and the first part is proved.

(ii) Since $\mu_{0}=0$, it follows that $Q_{j}(0)=1$. For $v \leq 0, Q_{j}(v) \geq Q_{j}(0)=1$, we get

$$
\sum_{j=0}^{\infty} \frac{1}{\lambda_{j} \pi_{j} Q_{j}(v) Q_{j+1}(v)} \leq \sum_{j=0}^{\infty} \frac{1}{\lambda_{j} \pi_{j}}<\infty
$$

and

$$
\infty=\sum_{j=0}^{\infty} \pi_{j} \leq \sum_{j=0}^{\infty} \pi_{j} Q_{j}^{2}(v)
$$

which finishes the proof.

Remark 13 Suppose (12) holds. Then for each process $\widetilde{\mathcal{X}}^{(x, v)}$ (mentioned in Theorem 11), $0<x \leq 1 / S^{(v)}, v \leq \xi_{1}, v$-similar to the process $\mathcal{X}$, if

$$
\sum_{j=0}^{\infty} \frac{1}{\lambda_{j} \pi_{j} Q_{j}(v) Q_{j+1}(v)\left(1-x S_{j}^{(v)}\right)\left(1-x S_{j-1}^{(v)}\right)} \sum_{i=0}^{j} \pi_{i} Q_{i}^{2}(v)\left(1-x S_{i-1}^{(v)}\right)^{2}=\infty
$$

and

$$
\sum_{j=0}^{\infty} \frac{1}{\lambda_{j} \pi_{j} Q_{j}(v) Q_{j+1}(v)\left(1-x S_{j}^{(v)}\right)\left(1-x S_{j-1}^{(v)}\right)} \sum_{i=j+1}^{\infty} \pi_{i} Q_{i}^{2}(v)\left(1-x S_{i-1}^{(v)}\right)^{2}=\infty,
$$

its limiting conditional distribution and the doubly limiting conditional distribution are given by

$$
\lim _{t \rightarrow \infty} \tilde{r}_{i j}(t)=\frac{\pi_{j} Q_{j}(v) Q_{j}\left(\xi_{1}\right)\left(1-x S_{j-1}^{(\nu)}\right)}{\sum_{k=0}^{\infty} \pi_{k} Q_{k}(v) Q_{k}\left(\xi_{1}\right)\left(1-x S_{k-1}^{(v)}\right)}, \quad \lim _{t \rightarrow \infty} \lim _{s \rightarrow \infty} \tilde{r}_{i j}(t, s)=\frac{\pi_{j} Q_{j}^{2}\left(\xi_{1}\right)}{\sum_{k=0}^{\infty} \pi_{k} Q_{k}^{2}\left(\xi_{1}\right)} .
$$


Remark 14 Let us notice that according to Theorem 9 there exists a unique birth-death process $\tilde{\mathcal{X}}^{(v)}$ with $\tilde{\mu}_{0}=0$ which is $v$-similar to the process $\mathcal{X}$. For such process $\widetilde{\mathcal{X}}^{(v)}$ if

$$
\sum_{j=0}^{\infty} \pi_{j} Q_{j}^{2}(v)=\infty
$$

and

$$
\sum_{j=0}^{\infty} \frac{1}{\lambda_{j} \pi_{j} Q_{j}(v) Q_{j+1}(v)} \sum_{i=0}^{j} \pi_{i} Q_{i}^{2}(v)=\infty
$$

then its limiting conditional distribution and the doubly limiting conditional distribution are given by

$$
\lim _{t \rightarrow \infty} \tilde{r}_{i j}(t)=\frac{a_{j} \pi_{j} Q_{j}(v) Q_{j}\left(\xi_{1}\right)}{\sum_{k=0}^{\infty} a_{k} \pi_{k} Q_{k}(v) Q_{k}\left(\xi_{1}\right)},
$$

where

$$
a_{j}=\frac{\int_{v}^{\infty}(\sigma-v)^{-1} Q_{i}(\sigma) d \varrho(\sigma)}{Q_{i}(v) \int_{v}^{\infty}(\sigma-v)^{-1} d \varrho(\sigma)}
$$

and

$$
\lim _{t \rightarrow \infty} \lim _{s \rightarrow \infty} \tilde{r}_{i j}(t, s)=\frac{\pi_{j} Q_{j}^{2}\left(\xi_{1}\right)}{\sum_{k=0}^{\infty} \pi_{k} Q_{k}^{2}\left(\xi_{1}\right)} .
$$

Example 15 Let us consider a birth-death process $\mathcal{X}$ with constant parameters $\lambda_{n}=\lambda$, $\mu_{n}=\mu$ for $n \in \mathcal{N}$. In this case the birth-death polynomials are of the form

$$
Q_{n}(x)=\left(\frac{\mu}{\lambda}\right)^{\frac{n}{2}} U_{n}\left(\frac{\lambda+\mu-x}{2 \sqrt{\lambda \mu}}\right), \quad n \geq 0,
$$

where $U_{n}(x)$ denote the Chebyshev polynomials of the second kind. In such a situation $\xi_{1}=\inf (\operatorname{supp}(\varrho))=\lambda+\mu-2 \sqrt{\lambda \mu}$. The corresponding measure $\varrho$ satisfies

$$
d \varrho(x)=\frac{1}{2 \pi \lambda \mu} \sqrt{4 \lambda \mu-(\lambda+\mu-x)^{2}} d x
$$

in the interval $|\lambda+\mu-x|<2 \sqrt{\lambda \mu}$, and it is zero outside this interval. For $\mu>\lambda$ the limiting and doubly limiting distributions of the process $\mathcal{X}$ equal

$$
\begin{aligned}
& \lim _{t \rightarrow \infty} r_{i j}(t)=\left(\sqrt{\frac{\lambda}{\mu}}-1\right)^{2}\left(\frac{\lambda}{\mu}\right)^{\frac{j}{2}}(j+1), \\
& \lim _{t \rightarrow \infty} \lim _{s \rightarrow \infty} r_{i j}(t, s)=0 .
\end{aligned}
$$

It is obvious that for $\lambda \geq \mu$ both distributions of $\mathcal{X}$ equal zero. 
We describe the one-parameter family $\left\{\widetilde{\mathcal{X}}^{(x, v)}\right\}$ of processes $v$-similar to $\mathcal{X}$. Let $v=\lambda+$ $\mu-2 b \sqrt{\lambda \mu} \leq \xi_{1}$, where $b \geq 1$. We get $Q_{n}(v)=\left(\frac{\mu}{\lambda}\right)^{n / 2} U_{n}(b)$. According to Theorem 11 we have

$$
S_{n}^{(v)}=\left(\lambda_{0}+\mu_{0}-v\right) \sum_{k=0}^{n} \frac{1}{\lambda_{k} \pi_{k} Q_{k}(v) Q_{k+1}(v)}=2 b \sum_{k=0}^{n} \frac{1}{U_{k}(b) U_{k+1}(b)} .
$$

Using the well-known properties of the Chebyshev polynomials of the second kind, i.e.

$$
U_{n}(x) U_{n+2}(x)+1=U_{n+1}^{2}(x)
$$

and

$$
U_{n}(x)=\frac{\left(x+\sqrt{x^{2}-1}\right)^{n+1}-\left(x-\sqrt{x^{2}-1}\right)^{n+1}}{2 \sqrt{x^{2}-1}}
$$

we can prove that

$$
S_{n}^{(v)}=2 b \sum_{k=0}^{n} \frac{1}{U_{k}(b) U_{k+1}(b)}=2 b \frac{U_{n}(b)}{U_{n+1}(b)}
$$

and

$$
S^{(v)}=\lim _{n \rightarrow \infty} S_{n}^{(v)}=2 b\left(b-\sqrt{b^{2}-1}\right) .
$$

Additionally let us notice that

$$
\sum_{n=0}^{\infty} \pi_{n} Q_{n}^{2}(v)=\sum_{n=0}^{\infty} U_{n}^{2}(b)=\infty
$$

Hence any member of the family

$$
\left\{\widetilde{\mathcal{X}}^{(x, v)}: 0 \leq x \leq \frac{b+\sqrt{b^{2}-1}}{2 b}, v=\lambda+\mu-2 b \sqrt{\lambda \mu}\right\}
$$

with parameters

$$
\begin{aligned}
& \tilde{\lambda}_{0}^{(x, v)}=\sqrt{\lambda \mu} \cdot \frac{U_{1}(b)-2 b x U_{0}(b)}{U_{0}(b)}, \\
& \tilde{\lambda}_{n}^{(x, v)}=\sqrt{\lambda \mu} \cdot \frac{U_{n+1}(b)-2 b x U_{n}(b)}{U_{n}(b)-2 b x U_{n-1}(b)}, \\
& \tilde{\mu}_{n+1}^{(x, \nu)}=\sqrt{\lambda \mu} \cdot \frac{U_{n}(b)-2 b x U_{n-1}(b)}{U_{n+1}(b)-2 b x U_{n}(b)}, \\
& \tilde{\mu}_{0}^{(x, v)}=2 b x \sqrt{\lambda \mu}
\end{aligned}
$$

is $v$-similar to the process $\mathcal{X}$. In Figure 1 we show graphs of $\tilde{\lambda}_{n}^{(x, v)}$ for $n=0,1,2,3,4$ in the case $\lambda=\frac{1}{7}, \mu=\frac{1}{5}, b=2$. 


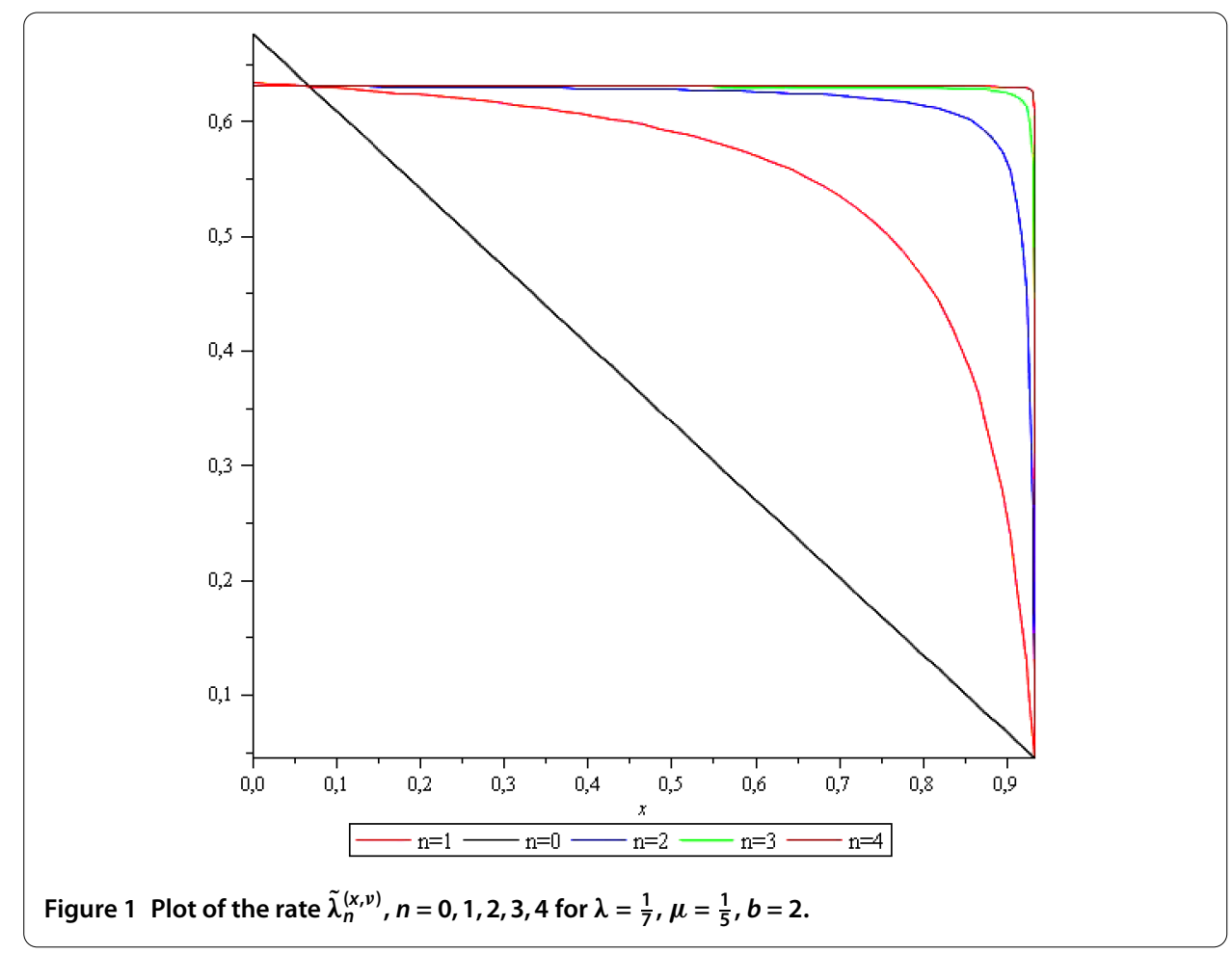

Let us consider $b=1$, then the family $\widetilde{\mathcal{X}}^{(x, v)}$ takes the form $\left\{\widetilde{\mathcal{X}}^{(x, v)}: 0 \leq x \leq 1 / 2, v=\lambda+\right.$ $\mu-2 \sqrt{\lambda \mu}$. The series $B, C$, and $D$ are divergent for the whole family. For $x=0$ we get the birth-death process $\tilde{\mathcal{X}}^{(v)}$ with $\tilde{\mu}_{0}^{(v)}=0$ and

$$
a_{n}=\frac{\int_{-1}^{1} U_{n}(\sigma) \sqrt{1-\sigma^{2}}(1-\sigma)^{-1} d \sigma}{U_{n}(1) \int_{-1}^{1} \sqrt{1-\sigma^{2}}(1-\sigma)^{-1} d \sigma}=\frac{1}{U_{n}(1)}=\frac{1}{n+1},
$$

where it is easily proved by induction that $\int_{-1}^{1} U_{n}(\sigma) \sqrt{1-\sigma^{2}}(1-\sigma)^{-1} d \sigma=\pi$ for $n \geq 0$.

Therefore

$$
\lim _{t \rightarrow \infty} \tilde{r}_{i j}^{(v)}(t)=\frac{(j+1) \int_{-1}^{1} U_{j}(\sigma) \sqrt{1-\sigma^{2}}(1-\sigma)^{-1} d \sigma}{\sum_{k=0}^{\infty}(k+1) \int_{-1}^{1} U_{k}(\sigma) \sqrt{1-\sigma^{2}}(1-\sigma)^{-1} d \sigma}=0 .
$$

For $0<x \leq 1 / 2$ we have the processes $\tilde{\mathcal{X}}^{(x, v)}$ with $\tilde{\mu}_{0}^{(x, v)}>0$ and

$$
\lim _{t \rightarrow \infty} \tilde{r}_{i j}^{(x, v)}(t)=\frac{(j+1)\left(U_{j}(1)-2 x U_{j-1}(1)\right)}{\sum_{k=0}^{\infty}(k+1)\left(U_{k}(1)-2 x U_{k-1}(1)\right)}=0 .
$$

For all processes $\widetilde{\mathcal{X}}^{(x, v)}, 0 \leq x \leq 1 / 2$, we get

$$
\lim _{t \rightarrow \infty} \lim _{s \rightarrow \infty} \tilde{r}_{i j}^{(x, v)}(t, s)=\frac{(j+1)}{\sum_{k=0}^{\infty}(k+1)^{2}}=0 .
$$


Authors' contributions

The authors declare that the study was realized in collaboration with the same responsibility. All authors read and approved the final manuscript.

\section{Acknowledgements}

This work is supported by Białystok University of Technology (Grant No. S/WI/2/2011).

Received: 13 February 2015 Accepted: 1 July 2015 Published online: 13 August 2015

\section{References}

1. Di Crescenzo, A: On some transformation properties of birth-and-death processes. In: Trappl, R (ed.) Cybernetics and Systems '94, pp. 839-846. World Scientific, Singapore (1994)

2. Lenin, RB, Parthasarathy, PR, Scheinhardt, WR, van Doorn, EA: Families of birth-death processes with similar time-dependent behaviour. J. Appl. Probab. 37, 835-849 (2000)

3. Pollett, PK: Similar Markov chains. J. Appl. Probab. 38A, 53-65 (2001)

4. Schiefermayr, K: Random walks with similar transition probabilities. J. Comput. Appl. Math. 153(1-2), 423-432 (2003)

5. Di Crescenzo, A, Martinucci, B: A first-passage-time problem for symmetric and similar two-dimensional birth-death processes. Stoch. Models 24(3), 451-469 (2008)

6. van Doorn, EA: Conditions for the existence of quasi-stationary distributions for birth-death processes with killing Stoch. Process. Appl. 122(6), 2400-2410 (2012)

7. van Doorn, EA, Zeifman, Al: Birth-death processes with killing. Stat. Probab. Lett. 72(1), 33-42 (2005)

8. van Doorn, EA, Zeifman, Al: Extinction probability in a birth-death process with killing. J. Appl. Probab. 42(1), 185-198 (2005)

9. Callaert, H, Keilson, J: On exponential ergodicity and spectral structure for birth-death processes I. Stoch. Process. Appl. 1, 187-216 (1973)

10. Callaert, H, Keilson, J: On exponential ergodicity and spectral structure for birth-death processes II. Stoch. Process. Appl. 1, 217-235 (1973)

11. Di Crescenzo, A, Martinucci, B: On a symmetric, nonlinear birth-death process with bimodal transition probabilities. Symmetry 1(2), 201-214 (2009)

12. Schoutens, W: Birth and death processes, orthogonal polynomials and limiting conditional distributions. Math. Sci. 25, 87-93 (2000)

13. Karlin, S, McGregor, JL: The differential equations of birth-and-death processes, and the Stieltjes moment problem. Trans. Am. Math. Soc. 85, 589-646 (1957)

14. Chihara, TS: An Introduction to Orthogonal Polynomials. Gordon \& Breach, New York (1978)

15. Anderson, WJ: Continuous-Time Markov Chains: An Applications-Oriented Approach. Springer, New York (1991)

16. Kijima, M, Nair, MG, Pollett, PK, van Doorn, EA: Limiting conditional distributions for birth-death processes. Adv. Appl. Probab. 29(1), 185-204 (1997)

17. van Doorn, EA: Birth-death processes and associated polynomials. J. Comput. Appl. Math. 153, 497-506 (2003)

\section{Submit your manuscript to a SpringerOpen ${ }^{\circ}$ journal and benefit from:}

- Convenient online submission

Rigorous peer review

- Immediate publication on acceptance

- Open access: articles freely available online

- High visibility within the field

- Retaining the copyright to your article 